\title{
Outdoor education in Aotearoa New Zealand: A new vision for the twenty first century
}

\author{
Reviewed by Peter Martin \\ University of Ballart
}

Irwin,D., Straker, J. \& Hill, A. (Eds.). (2012). Outdoor Education in Aotearoa New Zealand: A new Vision for the Twenty First Century Christchurch: CPIT

ISBN 978-0-908668-12-0

The purpose of this work, as outlined by the editors in their opening chapter is "to provide a practical, insightful and innovative reappraisal of outdoor education theory and practice" (p.13). Big claim, big task. Each reader will no doubt form their own view as whether these challenges are met. Largely this will depend on the reader's own background, beliefs and practices of outdoor education -"alternative visions" (p. 14) assume a common starting point.

What the book does well is pull together ideas about outdoor education and examines these in light of contemporary educational, social and environmental thinking. The book is an edited series of chapters from well-known New Zealand academics and outdoor educators. The contributions vary in focus and academic analysis. This is good - it provides differing readership with plenty to chew on.

The new vision in this collective work is that of outdoor education based upon a socio-ecological perspective. Such a perspective challenges the traditions and basis of outdoor education in adventure pursuits and renders it inherently political. As a New Zealand publication this also foregrounds the importance of Maori culture, and such perspectives are prominent throughout.

Outdoor Education, despite its diversity of interpretations and defiance of definition does have a sense of history and practice. Perhaps, in work that seeks to critically examine and shift outdoor education practices, we might expect some overshoot or false lead. For me, some of the examples read more like geography excursions rediscovered, than a new vision for outdoor education in schooling. Some of this arises from the broader embracing Kiwi notion of education outside the classroom (EOTC) as a term more inclusive of a range of curricula than outdoor education might be in other countries.

That said, there is much in this work that encourages the reader to carefully scrutinise existing sometimes taken for granted assumptions about what outdoor education can contribute to schooling. There are some excellent examples of innovative teaching, linked to local context and culture. Maintaining integrity of a book that advocates a more place based approach to outdoor education rightly means that much material is specific to New Zealand context, sometimes specific sites, but it is written thickly enough for the reader to make their own judgements about application to other settings.

Overall I really liked this book. There is a risk in a review of an edited work to highlight one contribution over another - and there were many that expressed well how educational experiences can contribute to ameliorating environmental and social challenges confronting western cultures into the 21st Century, however. The next to last chapter relates a slow journey over the Southern Alps, a trip of four days done in eleven. It acts as a fitting summary of the book by illustrating how the leader's outdoor wisdom, deep personal affinity and love of the outdoors, blended with critical educational insight informed by scholarship, can produce an educational experience that I felt confident was highly significant for the young people who participated. Would this slow journey have occurred a decade ago and been celebrated, I doubt it. This then, is the new vision and it shouldn't remain new for long.

\section{Abour the Reviewer}

Associate Professor Peter Martin (PhD) is Deputy Dean of Health Sciences at the University of Ballarat, Australia. He has a teaching and research interest in socio-critical outdoor education and development of human to nature relationships through outdoor education.

Email: peter.martin@ballarat.edu.au 